\title{
Hérnia de hiato deslizante em um felino persa: Relato de caso
}

\author{
Sliding hiatus hernia in persian feline: Case report \\ Hernia de hiato deslizante en un felino persa: Reporte de un caso
}

Patrick Willian Fischer Marques

ORCID: https://orcid.org/0000-0001-9937-3170 Centro Universitário Ingá, Brasil

E-mail: ra14502.16@uninga.edu.br

Sheila Dayane Rodrigues da Silva

ORCID: https://orcid.org/0000-0003-3101-4467 Centro Universitário Ingá, Brasil

E-mail: ra16271.17@uninga.edu.br Juliana Dotto do Amaral

ORCID: https://orcid.org/0000-0003-0328-7353 Centro Universitário Ingá, Brasil

E-mail: julianadotto13@gmail.com

Leonardo Martins Leal

ORCID: https://orcid.org/0000-0002-7864-6333 Centro Universitário Ingá, Brasil

E-mail: prof.leonardoleal@uninga.edu.br

Isaac Romani

ORCID: https://orcid.org/0000-0003-2128-8915 Centro Universitário Ingá, Brasil

E-mail: prof.isaacromani@uninga.edu.br

Gabriela Maria Benedetti Vasques

ORCID: https://orcid.org/0000-0001-9062-2230

Centro Universitário Ingá, Brasil

E-mail: prof.gabrielavasques@uninga.edu.br

\section{Resumo}

As hérnias de hiato deslizantes são incomuns em cães e gatos, apesar de serem frequentemente descritas em humanos. Elas podem ser classificadas em quatro tipos, sendo a hérnia de hiato deslizante a mais prevalente em pequenos animais e em animais braquicefálicos. Esta afecção pode, frequentemente, levar à regurgitação e pneumonia aspirativa, mas também pode ser assintomática. O objetivo deste relato é descrever um caso de hérnia de hiato deslizante em um felino persa, de seis meses de idade, com regurgitação após estresse. O paciente foi submetido a exame radiográfico simples, que não evidenciou alterações, e radiografia contrastada, na qual foi possível identificar a protusão do estômago através da junção gastroesofágica. O tratamento instituído foi anti-inflamatório, antiácido, e fracionamento da ingesta alimentar, visando a recuperação da esofagite e diminuição do tempo de esvaziamento gástrico. Seis meses após o diagnóstico o animal não manifestava mais sintomatologia clínica, e em novo exame de imagem, não apresentou protrusão do estômago concluindo assim a eficácia no tratamento medicamentoso e a importância da radiografia contrastada para o diagnóstico.

Palavras-chave: Esôfago; Esofagograma; Radiografia; Refluxo.

\begin{abstract}
Sliding hiatus hernias are uncommon in dogs and cats, although they are often described in humans. They can be classified into four types, with sliding hiatus hernia being the most prevalent in small animals. This condition can often lead to regurgitation and aspiration pneumonia, but it can also be asymptomatic. The purpose of this report is to describe a case of sliding hiatus hernia in a six-month-old Persian cat with regurgitation after stress. The patient was submitted to a simple radiographic exam, which showed no alterations, and contrasted radiography, in which it was possible to identify the protrusion of the stomach through the gastroesophageal junction. The treatment instituted was anti-inflammatory, antacid, and fractionation of food intake, aiming at the recovery of esophagitis and reducing gastric emptying time. Six months after diagnosis, the animal had no more clinical symptoms, and in a new imaging exam, it did not show protrusion of the stomach, thus concluding the efficacy in drug treatment and the importance of the contrasted radiography for diagnosis.
\end{abstract}

Keywords: Esophagus; Esophagogram; Radiography; Regurgitation. 


\begin{abstract}
Resumen
Las hernias de hiato deslizantes son inusuales en perros y gatos, aunque a menudo se describen en humanos. Se pueden clasificar en cuatro tipos, siendo la hernia de hiato deslizante la más prevalente en animales pequenos y en animales braquicéfalos. Esta afección a menudo puede conducir a la regurgitación y neumonía por aspiracíon, pero también puede ser asintomática. El objetivo de este reporte es describir un caso de hernia de hiato deslizante en un felino persa, de seis meses de edad, con regurgitacíon después del estrés. El paciente fue sometido a un examen radiográfico simple, que no mostró alteraciones, y a una radiografía contrastada, en la que se pudo identificar la protusíon del estómago a través de la unión gastroesofágica. El tratamiento administrado fue antiinflamatorio, antiácido, y fraccionamiento de la ingesta alimentaria, centrando-se em la recuperacíon de la esofagitis y disminuición del tiempo de vaciamiento gástrico. Seis meses después del diagnóstico, el animal ya no manifestaba síntomas clínicos, y en un nuevo examen imagenológico, no presentó protusión del estómago, concluyendo así la eficacia en el tratamiento medicamentoso y la importancia de la radiografía contrastada para el diagnóstico.
\end{abstract}

Palabras clave: Esófago; Esofagograma; Radiografía; Reflujo.

\title{
1. Introdução
}

Hérnia hiatal é incomum em cães e gatos (Bright, Sackman, DeNovo \& Toal, 1990; Lorinson \& Bright, 1998) embora seja bem reconhecida em humanos. São causadas por anomalias congênitas ou adquiridas no hiato esofágico, que está ligado ao esôfago por uma membrana gastroesofágica e, em condições normais, permite apenas um pequeno movimento da parte abdominal do esôfago para o tórax (Sivacolundhu, Read \& Marchevsky, 2002).

Hérnias de hiato são formadas pela protusão da víscera abdominal através do hiato esofágico, podendo resultar em refluxo e regurgitação, e posteriormente esofagite e megaesôfago (Miles, Pope \& Jergens, 1988). Sivacolundhu et al. (2002) classificam as hérnias em quatro tipos, sendo hérnia de hiato deslizante, hérnia de hiato periesofágica, hérnia diafragmática e intussuscepção gastroesofágica, derivando todas da redução do tônus do esfíncter caudal do esôfago.

Conforme Phillips et al. (2014) citam que animais braquicefálicos são os mais acometidos por hérnias de hiato e que a ocorrência mais frequente é na forma deslizante, também conhecida por axial, onde ocorre deslocamento simples do esôfago terminal, da junção gastroesofágica, e do estômago em parte cranial ao hiato. Além disso, e, Aslanian; Sharp e Garneau (2014) complementam dizendo que nesses animais o aumento da pressão intratorácica pode causar o deslizamento do conteúdo abdominal para o hiato esofágico. Mayhew et al. (2021) relatam em seu estudo realizado com cães braquicefálicos a ocorrência comum de hernia de hiato deslizante associada a refluxo gastroesofágico, e dentre as complicações citam esofagite, estenose esofágica, regurgitação de sólidos e líquidos, e pneumonia aspirativa.

Pequenos animais podem apresentar quadros severos de regurgitação, vômito e problemas respiratórios associados à pneumonia aspirativa (Sivacolundhu et al., 2002), no entanto, alguns animais são assintomáticos, como no relato de Santos L. e Santos G. (2016) onde o paciente não apresentava sinais clínicos expressivos e foi diagnosticado com hérnia de hiato após o tutor buscar atendimento tendo como queixa principal a baixa ingestão de alimentos manifestada pelo animal.

Tratando-se de diagnóstico da doença, Sivacolundhu et al. (2002) entram em consenso sobre a dificuldade de observála em radiografia simples, mas não descartam sua possibilidade de visualização, exemplificando, Gaschen (2019), descreve que se há mistura de opacidade de tecidos moles e de gás na região topográfica de aorta e veia cava, pode-se sugerir o diagnóstico. Da Silva (2021) alerta que a hérnia do tipo deslizante pode ter caráter dinâmico e intermitente, o que pode ser um fator de dificuldade no diagnóstico radiográfico. Mitsuoka et al. (2002) confirmam a detecção de hérnias de hiato deslizante por esofagograma contrastado com bário, ou fluoroscopia contrastada, onde é possível a localização do esfíncter esofágico caudal em região cranial ao diafragma, ainda Broux et al. (2017) mostraram que a realização de uma pressão intra-abdominal e elevação da garupa durante a realização dos exames pode auxiliar na identificação da patologia.

A endoscopia é citada por Birchard e Sherding (2013) como outro meio de diagnóstico, pois torna possível localizar e visualizar a falha ou alargamento da membrana frenoesofágica. Reeve, Sutton, Friend e Warren-Smith (2017) em seu estudo evidenciou que a fluoroscopia tem eficácia relevante no diagnóstico das hérnias de hiato. 
O animal assintomático não requer tratamento, mas para os indivíduos sintomáticos o tratamento medicamentoso é indicado e envolve a utilização de sulcrafato, com a finalidade de criar uma camada protetora para úlceras esofágicas, e antiácido para neutralizar a acidez gástrica. A dieta alimentar deve ser associada ao tratamento objetivando que animais obesos percam peso, e o padrão alimentar deve ser baseado no fornecimento de pequenos alimentos com baixo teor de gordura, 3 a 4 vezes ao dia, para auxiliar o esvaziamento gástrico, reduzir o volume de secreção de ácidos digestivos, e diminuir o refluxo gastro-esofagico (Sivacolundhu et al., 2002).

O prognóstico para essa patologia depende da resposta medicamentosa do paciente e do grau da hérnia hiatal. Em animal que responde à terapia medicamentosa e não requer intervenção cirúrgica, o prognóstico é bom (Phillips et al., 2014).

Dada a escassez de relatos de casos abordando o tema em felinos, o objetivo deste trabalho é relatar um caso de hérnia de hiato deslizante em um gato da raça persa, de 6 meses.

\section{Metodologia}

Este relato é uma pesquisa descrita, de abordagem qualitativa, caracterizado pela coleta de dados direta, no ambiente natural, onde o pesquisador é o principal instrumento. A maioria dos dados coletados são descritivos e sua análise tende a seguir o processo hipotético indutivo (Pereira et al., 2018).

\section{Relato de caso}

Foi atendido em uma clínica veterinária escola da cidade de Maringá (Paraná, Brasil), um felino da raça persa, macho não castrado, com 6 meses, pesando 4 quilos. A queixa principal do tutor era a manifestação de regurgitação nos 4 dias que antecederam a consulta. O tutor relatava que os episódios aconteciam logo após o animal se alimentar, com aspecto de alimento não digerido, e segundo ele, observou que se iniciaram após um momento de brincadeira, na qual o animal se assustou e apresentou estresse respiratório.

O paciente possuía protocolo vacinal completo e atualizado, histórico recente de uso de antiparasitário, frequência cardíaca e respiratória dentro dos parâmetros da normalidade, apetite preservado denotado pelas tentativas de se alimentar, entretanto, havia regurgitação após a alimentação.

Sob suspeita de doença esofágica, o animal foi encaminhado ao exame radiográfico simples e contrastado (esôfagograma). Incialmente realizou-se radiografia simples em projeção lateral direita e ventrodorsal (Figura 1), onde não foram visualizadas quaisquer alterações em topografia de esôfago. Na sequência, foi administrado $10 \mathrm{ml}$ de sulfato de bário por via oral, e foram realizadas mais duas radiografias, nas mesmas projeções, feitas imediatamente após a administração do meio de contraste. Na radiografia contrastada, em projeção lateral direita (Figura 2) foi possível observar a protrusão do esôfago e estômago em porção dorsal do diafragma constatando a presença de hérnia de hiato, contudo, na projeção ventrodorsal a hérnia não foi visualizada. $\mathrm{O}$ exame de ultrassonografia abdominal também foi realizado, mas não indicou alterações dignas de nota. 
Research, Society and Development, v. 10, n. 6, e54510615984, 2021

(CC BY 4.0) | ISSN 2525-3409 | DOI: http://dx.doi.org/10.33448/rsd-v10i6.15984

Figura 1. Radiografia simples em projeção lateral direita sem alterações em topografia de esôfago.

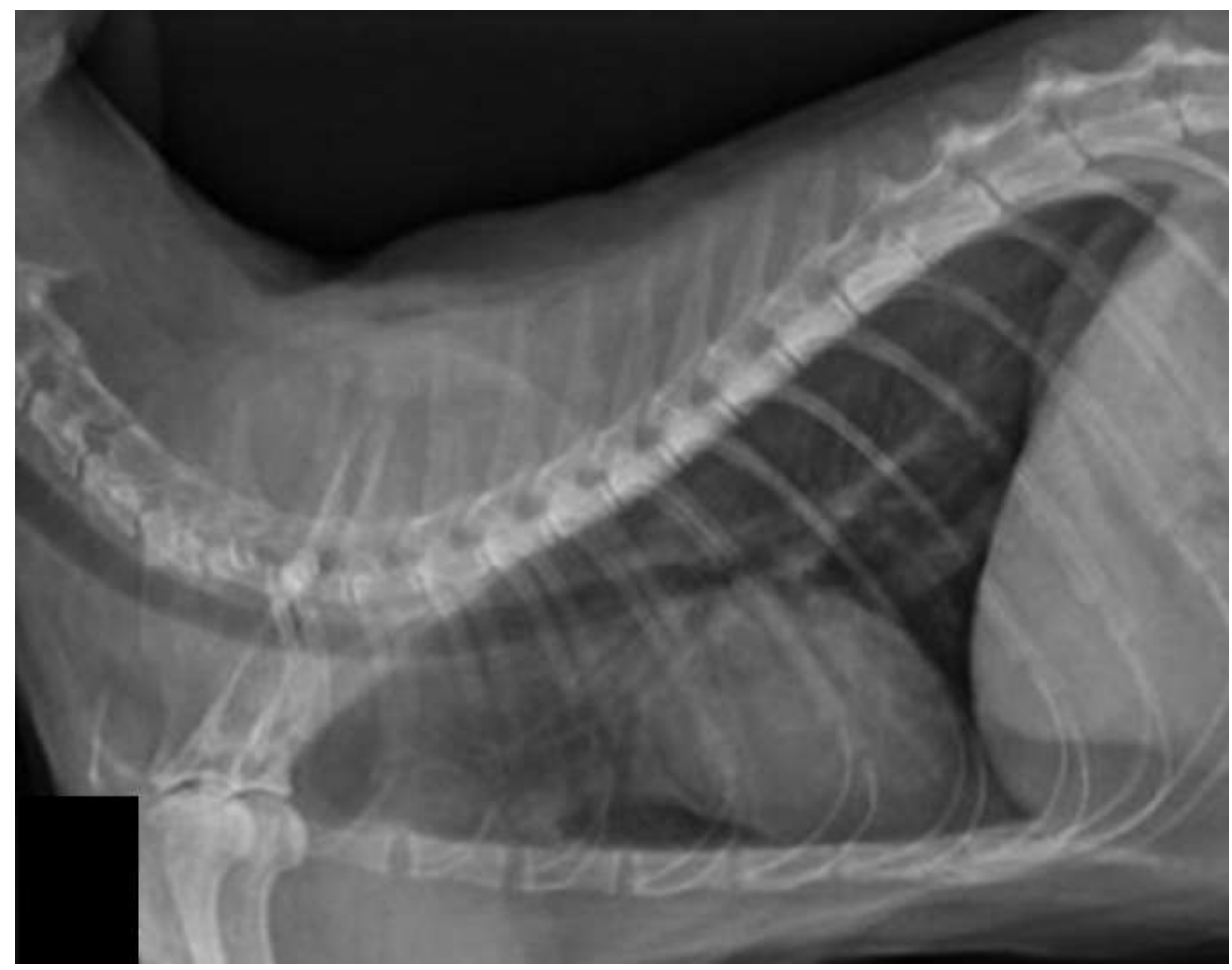

Fonte: Acervo dos autores.

Figura 2 - Radiografia contrastada evidenciando a protusão do esôfago (seta branca) em porção anatômica caudal do tórax.

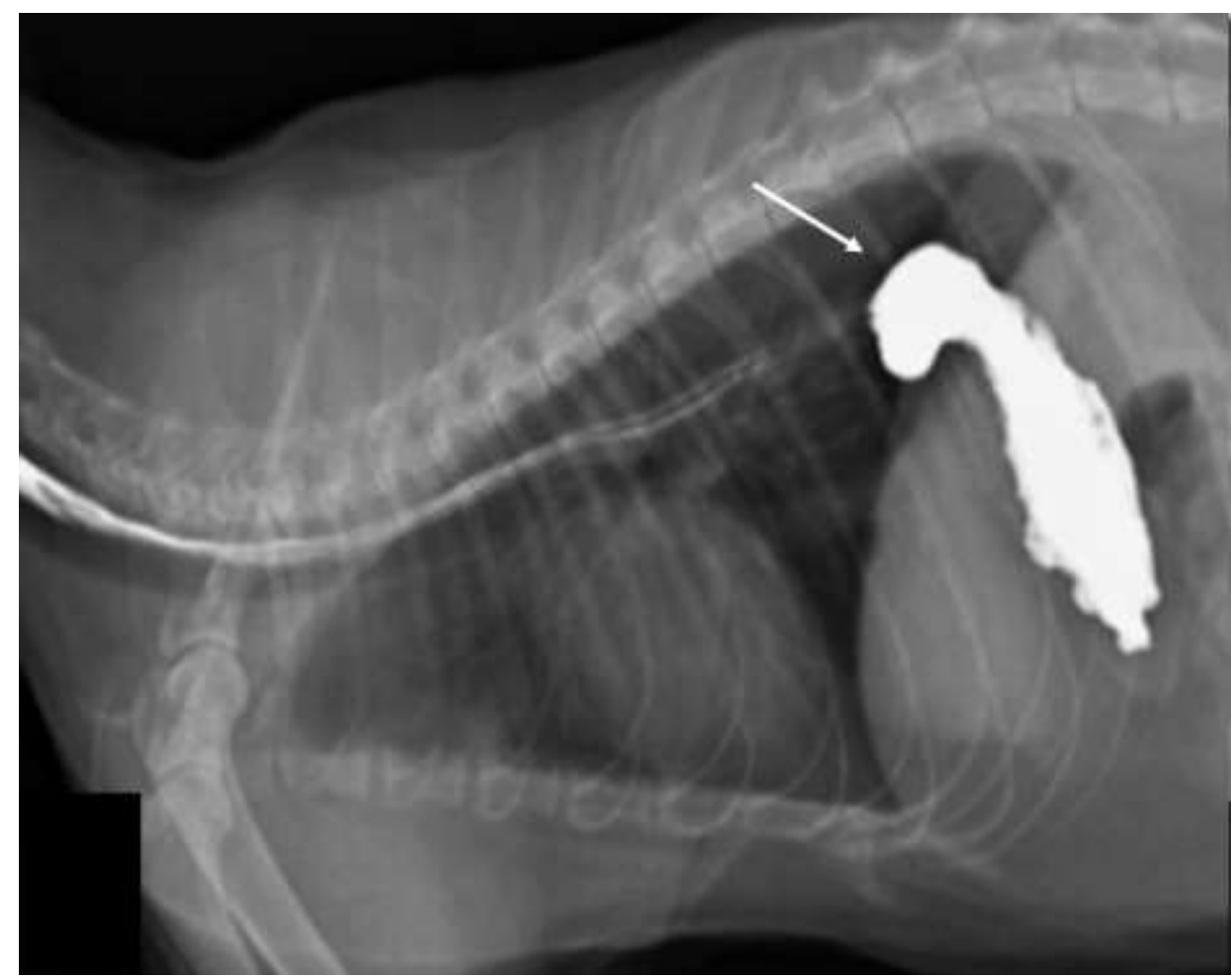

Fonte: acervo dos autores. 
Os achados radiográficos apontaram para o diagnóstico sugestivo de hérnia de hiato, face a isso, foi prescrito terapia medicamentosa baseada na utilização de meloxicam oral, na dose de $0,01 \mathrm{mg} / \mathrm{kg}$, a cada 24 horas, durante 5 dias, sucralfato 0,5mg a cada 12 horas, durante 7 dias. Além disso, recomendou-se o fracionamento da ingesta em até 4 vezes ao dia.

O paciente retornou seis meses após a primeira avaliação, onde o tutor relatou que todos os sinais clínicos haviam cessado, estando o animal com ingestão normal de alimentos sólidos e água, e permanecendo a ingesta fracionada como recomendado. No novo exame clínico não foram observadas alterações diferentes da normalidade.

Com o objetivo de reavaliar a hérnia hiatal, realizou-se um novo exame radiográfico, com as mesmas etapas do realizado na primeira consulta, como descrito acima, sendo o primeiro exame simples e o segundo contrastado. Nestes exames não se observou a protusão do estômago pela junção gastroesofágica, estando todas as projeções dentro da normalidade. Com isso, estabeleceu-se o diagnóstico de hérnia de hiato deslizante.

\section{Discussão}

Hérnia de hiato deslizante é o tipo mais comum em cães (Sivacolundhu et al., 2002), entretanto em gatos há poucos casos descritos, o que ressalta a relevância do uso de exames de imagem para a investigação de alterações esofágicas.

As hérnias de hiato geralmente são congênitas em cães (75\%) e em gatos (67\%) e podem permanecerem assintomáticas ou demostrarem sinais clínicos com menos de 1 ano, o que levanta a hipótese desta afecção ter origem congênita no animal do relato, já que ele possuía 6 meses (Sivacolundhu et al., 2002).

Vários fatores contribuem para o surgimento dessa afecção, entre eles está a predisposição apresentada por animais braquicefálicos que frequentemente sofrem de dificuldade respiratória oriunda da sua conformação anatômica. Reeve et al. (2017) citam uma hipótese, na qual, o aumento da pressão entre o tórax e a cavidade abdominal acaba criando um gradiente que faz com que as estruturas se desloquem pela membrana gastroesofágica, hipótese também descrita por Broux et al. (2017). Sobre a hipótese de aumento de pressão intratorácica, em um estudo realizado por Hardie, Ramirez e Clary (1998), os autores correlacionam fraturas de costela e o esforço respiratório com as hérnias de hiato, além de também descreverem que cães braquicefálicos, pela anatomia do sistema respiratório, possuem maior incidência da afecção.

O paciente relatado é um felino da raça persa, por sua vez, braquicefálico, e seu diagnóstico corrobora com os resultados dos estudos (Sivacolundhu et al., 2002; Phillips et al., 2014) que indicam maior prevalência nesta classe. Entretanto, não há a descrição da raça em específico como mais susceptível às hérnias de hiato.

Outra hipótese levantada abrange a relação com o susto que o tutor relatou, e o aparecimento dos sinais clínicos. O animal pode ter tido um pico de estresse respiratório, e em conjunto com sua formação anatômica, levou a protusão do estômago para a cavidade torácica. Essa situação é semelhante ao caso de DeSandre-Robinson, Madden e Walker (2011), onde um felino com estenose nasofaríngea apresentou, logo após esforço respiratório, hérnia de hiato.

Refluxos são as manifestações clínicas mais comuns em casos de hérnia de hiato, já que qualquer alteração no esfíncter esofágico pode ocasioná-los (Gambino et al., 2015). Ainda que no caso relatado o único sinal clínico observado tenha sido a ocorrência dos refluxos após a alimentação, há muitos casos na literatura que associam quadros respiratórios como sinal da afecção. Ilustrando o exposto, Keeley, Puggioni e Pratschke (2008) relatam um canino da raça pug, que apresentava dispneia aguda após alimentação e, após investigação, constataram a presença da hérnia de hiato, reforçando que as manifestações podem variar entre espécies e raças.

Entre os acometimentos respiratórios, a pneumonia aspirativa se destaca como preocupação principal, devido a sua frequente ocorrência em quadros de hérnia hiatal (Sivacolundhu et al., 2002; Phillips et al., 2014). Presume-se que o curto período transcorrido desde o início da sintomatologia até a procura de atendimento veterinário, foi um fator que pode justificar 
o não desenvolvimento de complicações respiratórias, uma vez que quanto menor o período em que o paciente sofrer com episódios de refluxo, menores serão suas chances de apresentar um quadro de pneumonia aspirativa.

O exame radiográfico é usualmente utilizado para diagnosticar casos de hérnias hiatais, porém, resultados falsonegativos podem acontecer, principalmente em casos de hérnias de hiato deslizantes (Phillips et al., 2014). No felino em questão, em projeções simples, não foi possível evidenciar nenhuma alteração. Apenas após a administração do sulfato de bário se visualizou o deslocamento cranial do estômago. Alguns autores (Owen, Morris \& Bateman, 2005) relatam que o uso de contraste nem sempre é necessário, principalmente se associadas com outras afecções, como a pneumonia aspirativa.

O esofagograma é o método de diagnóstico padrão ouro no diagnóstico, na análise de 31 casos feita por Phillips et al. (2014), essa técnica teve eficiência de $100 \%$ no diagnóstico das hérnias de hiato. O esofagograma neste caso foi eficiente e confirmou o diagnóstico.

O tratamento medicamentoso, em casos de hérnia de hiato deslizante, consiste principalmente em anti-inflamatórios, relacionando a possível esofagite causada pela protusão e antiácidos. Meloxicam e sucralfato foram administrados ao felino do relato e mostraram-se eficazes, já que o animal não apresentou novos episódios de regurgitação.

O fracionamento da dieta também é uma medida prescrita, já que possibilita a diminuição do tempo de esvaziamento gástrico, além de impedir o acúmulo de alimento no estômago, e eventual protusão pelo hiato. Esta medida foi instituída desde o diagnóstico até os seis meses subsequentes, e dessa forma o animal não apresentou mais sintomatologia. (Sivacolundhu $e t$ al., 2002) Hérnias de hiato do tipo deslizante assintomáticas não requerem tratamento cirúrgico, apenas cuidados com o peso do animal e dieta em pequenas porções 3 a 4 vezes ao dia (Sivacolundhu et al., 2002; Mayhew, Marks, Pollard, Culp, \& Kass 2017). Nesse caso se observa que a terapia medicamentosa teve sucesso nesse felino, que permaneceu sem sinais clínicos após o tratamento (Phillips et al., 2014). Em casos graves ou naqueles em que a conduta clínica não for suficiente para a remissão das manifestações, a intervenção cirúrgica com laparotomia exploratória e gastropexia costuma apresentar bons resultados quanto a melhora dos episódios de êmese e regurgitação, proporcionando maior qualidade de vida ao paciente (Silva T. 2021).

\section{Conclusão}

A anamnese e os sinais clínicos aliados aos exames de imagem foram significativos para o estabelecimento do diagnóstico de hérnia de hiato, e o tratamento medicamentoso instituído se mostrou eficaz. O esofagograma permitiu a identificação de sinais da hernia hiatal não visualizados na radiografia simples, solidificando a importância do uso da técnica contrastada. $\mathrm{O}$ relato de um felino da raça persa se mostra pertinente e corrobora com outros estudos relatando a ocorrência da afecção em braquicefálicos. Reforça-se com esse caso, a relevância da investigação de doenças esofágicas em gatos como emprego de exames de imagem. Para trabalhos futuros sugere-se estudos prospectivos em felinos com regurgitação empregando, principalmente, a fluoroscopia que permite a avaliação em tempo real da deglutição e caminho da ingesta pelo esôfago.

\section{Referências}

Aslanian, M. E., Sharp, C. R., \& Garneau, M. S. (2014). Gastric dilatation and volvulus in a brachycephalic dog with hiatal hernia. The Journal of small animal practice, 55(10), 535-537. https://doi.org/10.1111/jsap.12235

Birchard, S. J., \& Sherding, R. G. (2013). Doenças do esôfago e anormalidades de deglutição. In: R. M. Bright. (Ed.), Manual Saunders de clínica de pequenos animais. (3a ed., pp. 651-680). Roca.

Bright, R. M., Sackman, J. E., DeNovo, C., \& Toal, C. (1990), Hiatal hernia in the dog and cat: A retrospective study of 16 cases. Journal of Small Animal Practice, 31: 244-250. https://doi.org/10.1111/j.1748-5827.1990.tb00795.x

Broux, O., Clercx, C., Etienne, A. L., Busoni, V., Claeys, S., Hamaide, A., \& Billen, F. (2018). Effects of manipulations to detect sliding hiatal hernia in dogs with brachycephalic airway obstructive syndrome. Veterinary surgery : VS, 47(2), 243-251. https://doi.org/10.1111/vsu.12735 
Da Silva, T. R. F.(2021). Hérnia de hiato deslizante em cão: relato de caso. [Monografia de especialização, Universidade Federal do Rio Grande do Sul]. UFRGS Lume repositório digital. https://www.lume.ufrgs.br/handle/10183/219746

DeSandre-Robinson, D. M., Madden, S. N., \& Walker, J. T. (2011). Nasopharyngeal stenosis with concurrent hiatal hernia and megaesophagus in an 8-yearold cat. Journal of feline medicine and surgery, 13(6), 454-459. https://doi.org/10.1016/j.jfms.2011.01.007

Gambino, J. M., Sivacolundhu, R., DeLucia, M., \& Hiebert, E. (2015). Repair of a sliding (type I) hiatal hernia in a cat via herniorrhaphy, esophagoplasty and floppy Nissen fundoplication. JFMS open reports, 1(2), 2055116915602498. https://doi.org/10.1177/2055116915602498

Gaschen, L. (2019). O esôfago de cães e gatos. In: D.E. Thrall. (Ed.), Diagnóstico de Radiologia Veterinária. (7a ed., pp.1081-1176). Elsevier.

Hardie, E. M., Ramirez, O., 3rd, Clary, E. M., Kornegay, J. N., Correa, M. T., Feimster, R. A., \& Robertson, E. R. (1998). Abnormalities of the thoracic bellows: stress fractures of the ribs and hiatal hernia. Journal of veterinary internal medicine, 12(4), 279-287. https://doi.org/10.1111/j.19391676.1998.tb02123.x

Keeley, B., Puggioni, A. \& Pratschke, K. Congenital oesophageal hernia in a pug. Ir Vet J 61, 389 (2008). https://doi.org/10.1186/2046-0481-61-6-389

Lorinson, D., \& Bright, R. M. (1998). Long-term outcome of medical and surgical treatment of hiatal hernias in dogs and cats: 27 cases (1978-1996). Journal of the American Veterinary Medical Association, 213(3), 381-384.

Mayhew, P. D., Balsa, I. M., Marks, S. L., Pollard, R. E., Case. J. B., Culp, W. T. N., \& Giuffrida, M. A. (2021). Clinical and videofluoroscopic outcomes of laparoscopic treatment for sliding hiatal hernia and associated gastroesophageal reflux in brachycephalic dogs. Veterinary Surgery. 1-11. https://doi.org/10.1111/vsu. 13622

Mayhew, P. D., Marks, S. L., Pollard, R., Culp, W., \& Kass, P. H. (2017). Prospective evaluation of surgical management of sliding hiatal hernia and gastroesophageal reflux in dogs. Veterinary surgery : VS, 46(8), 1098-1109. https://doi.org/10.1111/vsu.12684

Miles, K. G., Pope, E. R., \& Jergens, A. E. (1988). Paraesophageal hiatal hernia and pyloric obstruction in a dog. Journal of the American Veterinary Medical Association, 193(11), 1437-1439.

Mitsuoka, K., Tanaka, R., Nagashima, Y., Hoshi, K., Matsumoto, H., \& Yamane, Y. (2002). Omental herniation through the esophageal hiatus in a cat. The Journal of veterinary medical science, 64(12), 1157-1159. https://doi.org/10.1292/jvms.64.1157

Owen, M. C., Morris, P. J., \& Bateman, R. S. (2005). Concurrent gastro-oesophageal intussusception, trichobezoar and hiatal hernia in a cat. New Zealand veterinary journal, 53(5), 371-374. https://doi.org/10.1080/00480169.2005.36579

Pereira, A. S., Shitsuka, D. M., Parreira, F. J., \& Shitsuka, R. (2018). Metodologia do trabalho científico. Ed. UAB/NTE/UFSM. https://www.ufsm.br/app/uploads/sites/358/2019/02/Metodologia-da-Pesquisa-Cientifica_final.pdf

Phillips, H., Corrie, J., Engel, D. M., Duffy, D. J., Holt, D. E., Kendall, A. R., Schmiedt, C. W., Vetter, A., Meren, I. L., Follette, C., Schaeffer, D. J., Mayhew, P. D., \& Marks, S. L. (2019). Clinical findings, diagnostic test results, and treatment outcome in cats with hiatal hernia: 31 cases (1995-2018). Journal of veterinary internal medicine, 33(5), 1970-1976. https://doi.org/10.1111/jvim.15583

Reeve, E. J., Sutton, D., Friend, E. J., \& Warren-Smith, C. (2017). Documenting the prevalence of hiatal hernia and oesophageal abnormalities in brachycephalic dogs using fluoroscopy. The Journal of small animal practice, 58(12), 703-708. https://doi.org/10.1111/jsap.12734

Santos, L. B., \& Santos, G. A. (2016) Evidenciação de hérnia de hiato em caninos por meio do gastrograma. Revista eletrônica da faculdade de ciências exatas e agrárias, 5(9), 35-40. https://docplayer.com.br/49228601-Evidenciacao-de-hernia-de-hiato-em-caninos-por-meio-do-gastrograma.html

Sivacolundhu, R. K., Read, R. A., \& Marchevsky, A. M. (2002). Hiatal hernia controversies--a review of pathophysiology and treatment options. Australian veterinary journal, 80(1-2), 48-53. https://doi.org/10.1111/j.1751-0813.2002.tb12046.x 